\title{
BMJ Open Impact of the COVID-19 pandemic on mental health of nursing students in Japan: protocol for a cross- sectional study
}

\author{
Jun Kako (D) , ${ }^{1}$ Yoshiyasu Ito, ${ }^{1}$ Kohei Kajiwara, ${ }^{2}$ Yasutaka Kimura, ${ }^{3}$ \\ Takahiro Kakeda, ${ }^{4}$ Seiji Hamanishi, ${ }^{4}$ Shinsuke Sasaki, ${ }^{5}$ Makoto Yamanaka, ${ }^{6}$ \\ Hana Kiyohara, ${ }^{1}$ Yuki Wakiguchi, ${ }^{1}$ Yoji Endo, ${ }^{4}$ Kimie Harada, ${ }^{2}$ Yuji Koga, ${ }^{7}$ \\ Michiko Ishida, ${ }^{7}$ Yoko Nishida, ${ }^{7}$ Masamitsu Kobayashi, ${ }^{8}$ Michihiro Tsubaki ${ }^{9}$
}

To cite: Kako J, Ito Y, Kajiwara K, et al. Impact of the COVID-19 pandemic on mental health of nursing students in Japan: protocol for a crosssectional study. BMJ Open 2021;11:e055916. doi:10.1136/ bmjopen-2021-055916

- Prepublication history for this paper is available online. To view these files, please visit the journal online (http://dx.doi org/10.1136/bmjopen-2021055916).

Received 27 July 2021 Accepted 09 November 2021

Check for updates

(c) Author(s) (or their employer(s)) 2021. Re-use permitted under CC BY-NC. No commercial re-use. See rights and permissions. Published by BMJ.

For numbered affiliations see end of article.

Correspondence to

Dr Jun Kako;

jun_kako@cnas.u-hyogo.ac.jp

\section{ABSTRACT}

Introduction The COVID-19 pandemic is spreading globally with a high risk of mortality. It is also significantly affecting mental health. For nursing students, the impact of COVID-19 on mental health is predicted to be significant; however, sufficient data have not been obtained. Therefore, this study will aim to assess the mental health of nursing students and evaluate the related factors.

Methods and analysis This proposed study is a crosssectional survey using a self-report questionnaire. An online questionnaire will be distributed among all nursing students of eight universities in Japan. The survey questionnaire will consist of questions related to demography, life satisfaction, fear of COVID-19, mental health and physical activities. The target sample size is 1300 nursing students. We will calculate descriptive statistics for each measurement item and perform univariate and logistic regression analyses to evaluate the potential risk factors for anxiety, depression and insomnia symptoms in nursing students. The strength of association will be assessed using the OR and its $95 \%$ Cls. Statistical significance will be set at a $p<0.05$.

Ethics and dissemination The protocol was approved by the Institutional Review Board (IRB) of the University of Hyogo on 22 March 2021 (ID: 2020F29). In addition, all of the participating facilities required ethical approval from their local IRBs. The findings will be disseminated through peer-reviewed publications and conference presentations. We believe that the proposed large-scale investigation of the mental health of nursing students during the COVID-19 pandemic and the relationship between mental health and fear of COVID-19 are novel and will be a strength of this study.

\section{INTRODUCTION}

An outbreak of COVID-19 began in Wuhan (Hubei Province, China) in December 2019 and has spread worldwide. As of 26 July 2021, 194080019 cases and 4162304 deaths have been reported worldwide as stated by WHO. ${ }^{1}$ In Japan, as of 27 July 2021, 875506 people have been infected and 15137 people have
Strengths and limitations of this study

- With the COVID-19 pandemic, evaluation of the mental health of nursing students, who are the future leaders of healthcare, is urgently warranted.

- A multi-institutional collaborative study is planned to obtain representative data from Japan, which is likely to be sufficient for international comparisons.

- Binary logistic regression analysis will be performed to identify factors associated with depression, anxiety and insomnia in nursing students.

- A limitation of this study is the expected low participation and response rates, which are inherent limitations of online surveys.

died. ${ }^{2}$ The massive outbreak of COVID-19 has increased the risk of death and has had a significant impact on the mental health. ${ }^{3-6}$

Mental health is a concept that includes emotional, psychological, and social wellbeing, and is considered to affect how we think, feel and act. $^{7}$ A systematic review of the prevalence of anxiety in the general population during the COVID-19 epidemic reported that $27 \%$ of the people had anxiety symptoms, with a particularly high prevalence in women and the elderly. Among healthcare workers with a reported prevalence of anxiety, depression, insomnia and stress in $30 \%-40 \%$ of the population, ${ }^{9-11}$ which is considered a public health problem. For nursing students who desire to become healthcare professionals in the future, mental health-related issues are even more complicated because such issues also directly affect their studies.

To prevent the spread of COVID-19, many countries have introduced social distancing and quarantine measures; as a result, globally, 31453440 students have been affected in some way in the context of their education as of 27 
July 2021, as reported by the UNESCO. ${ }^{12}$ Social distancing and quarantine measures have affected students' participation in face-to-face lectures and other learning-related activities for students. For students, participation in activities related to university is an important opportunity to maintain connections with classmates and faculty. Nursing students are missing out on hands-on training at hospitals and participation in hospital-sponsored internships due to COVID-19. The loss of such opportunities is a major obstacle for students ${ }^{13}$ and is predicted to affect the students' mental health. For example, in a recent study that was conducted among nursing students, investigating the impact of the COVID-19 pandemic, a low social presence and low satisfaction with remote education was reported. ${ }^{14}$ Another study conducted on nursing students reported on the development of an online environment and its impact on student satisfaction. ${ }^{15}$ Nursing students' satisfaction with their education and low social presence may have had an impact on their mental health.

During past epidemics such as SARS, it was reported that healthcare workers were at increased risk of burnout, secondary injuries, decreased job satisfaction, low morale and severe stress. ${ }^{16}$ Owing to COVID-19, nursing students are anxious about becoming a healthcare worker in the future. ${ }^{17}$ Anxiety is reported to be associated with the feelings of hopelessness, ${ }^{18}$ fear of COVID- $19^{19}$ and poor sleep quality. ${ }^{20}$ However, the scale of the COVID-19 pandemic is much larger than that of previous epidemics, and the demand for healthcare workers is expected to increase in the future. ${ }^{21-23}$ With this background, the mental health of nursing students who will become future healthcare workers can be an urgent public health concern. ${ }^{172}$ Although some universities have established health centres and have university staff with medical qualifications, to date, the impact of COVID-19 on the mental health of nursing students and evidence-based support have not been reported in a large scale, multiuniversity setting, indicating the need for further research and urgent solutions. ${ }^{25}$

In a previous study on nursing students during the COVID-19 pandemic, mental health-related symptoms, such as anxiety, depression, and stress, were investigated, and the prevalence of these symptoms was reported to be $30 \%-50 \% .^{26-29}$ Although this prevalence is higher than that in the general population ${ }^{30}$ and healthcare workers, ${ }^{9-11}$ studies on this subject are scarce and data are insufficient. Furthermore, these previous studies were relatively medium-sized, involving 300-1000 nursing students. Therefore, it would be desirable to conduct a study on a larger population to obtain more generalisable results. In addition, a previous study investigated the relationship between life satisfaction and anxiety, depression and stress symptoms among college students during the COVID-19 pandemic, and found that lower life satisfaction was associated with a lower behavioural ability to cope with mental health-related symptoms during the pandemic. ${ }^{31}$ Another study pointed out that high levels of physical activity among college students during the COVID-19 pandemic were associated with reduced anxiety and depression. ${ }^{32}$ However, the multifactorial assessment of mental health aspects, including the fear of COVID-19, has not been well investigated.

It is necessary to assess the level of mental health-related symptoms in nursing students and to analyse the potential risk factors associated with these symptoms to provide appropriate support. The understanding of the mental health of nursing students from the findings of this study may help educators create policies that will address the students' needs quickly and may also help improve the academic performance of nursing students.

\section{Aims}

The aim of this study is to evaluate the mental health of nursing students during COVID-19 outbreak. This study will describe the protocol developed to undertake this research.

The key research questions are:

1. What proportion of nursing students are screened as having moderate or higher anxiety, depression or insomnia when evaluated with the Generalised Anxiety Disorder-7 (GAD-7), ${ }^{33}$ Patient Health Questionnaire-9 $\left(\mathrm{PHQ}_{-}\right)^{34}$ and Insomnia Severity Index-7 (ISI-7) ${ }^{35}$ scales, respectively?

2. What is the level of fear of COVID-19 among nursing students when evaluated quantitatively using the Fear of COVID-19 scale (FCV-19S) ? ${ }^{36}$

3. What are the potential risk factors for anxiety, depression, and insomnia symptoms in nursing students?

4. Are there any correlations between anxiety, depression and insomnia symptoms in nursing students?

\section{METHODS AND ANALYSIS \\ Study design}

This is a cross-sectional survey targeting all nursing students in the selected institutions. The planned dates for data collection were between 16 August 2021 and 15 October 2021. In Japanese universities, late July to early August is often the period when grades for lecture courses are finalised, and unintended coercion may possibly be applied to gain cooperation during this period. Therefore, we will avoid this period when conducting the survey. For the selected institutions, we included both regions in Japan where COVID-19 is prevalent and regions where it is not (ie, is it an area where the Japanese government has declared a state of emergency or not?). The following eight universities in Japan were selected as the target institutions: University of Hyogo, Kansai University of School Welfare, Japanese Red Cross Kyushu International College of Nursing, Kawasaki University of Medical Welfare, Okayama Prefecture University, Meio University, Kitasato University Hospital and Aichi Medical University.

\section{Study procedure}

The survey will be a web-based questionnaire conducted using Microsoft Forms (Microsoft Office 365, USA). The explanatory document will be posted on the web page 
using the university web system or portal site system of the participating institution, or printed materials will be used to disclose information about this research. Reading the explanatory document, participants will be asked to click on the URL of the survey site, if they are interested to participate in the study. After checking the agreement column, the questionnaire will open. By checking the agreement column, the consent to participate in the study is deemed to have been obtained. In addition, it will be mentioned that the identity of participants cannot be revealed because this will be an anonymous questionnaire survey and that the answers cannot be withdrawn.

\section{Survey items}

In the questionnaire survey, we plan to inquire about background information (grade in school, age, sex and body mass index); changes in smoking, alcohol consumption and economic deprivation after the COVID-19 pandemic; living with family members; SARS-CoV-2 infection status of relatives and acquaintances; life satisfaction; mental health-related items and physical activities. Economic deprivation is asked in the following five cases: 'I became difficult,' 'I became a little difficult,' 'I remained the same,' 'I became a bit comfortable,' and 'I became comfortable'. Physical activities will be evaluated using the International Physical Activity QuestionnaireShort Form (IPAQ-SF). ${ }^{37}$ The Japanese version of the IPAQ-SF has been reported to have high reliability and validity ${ }^{38}$ with an intraclass correlation coefficient that ranges from 0.87 to 0.93 .

\section{Mental health-related items \\ Life satisfaction}

Life satisfaction will be evaluated using two parameters, 'current life satisfaction' and 'change in life satisfaction since the pandemic', with reference to the previous study by Tang et al. ${ }^{39}$ Current life satisfaction will be assessed using an 11-point Likert scale, ranging from ' 0 : extremely dissatisfied' to '10: extremely satisfied'. The change in life satisfaction after the pandemic will be asked to rate on a 5-point Likert scale of '-2: a lot worse', '-1: worse', '0: pretty much the same', ' 1 : better', and '2: a lot better'.

\section{Anxiety}

Anxiety will be evaluated using the GAD-7 scale. ${ }^{33}$ GAD-7 has been shown to have high sensitivity and specificity as a screening tool for GAD-7, with seven items on a 4-point scale, with a total score of 0-21. In this, 5-9, 10-14 and 15-21 points indicate mild, moderate, and severe level of symptoms, respectively. This scale had a good factorial validity and reliability. Several studies on mental health during the COVID-19 pandemic have used GAD-7..$^{10} 30$ The validity and utility of the Japanese version of GAD-7 have been confirmed. ${ }^{4041}$

\section{Depression}

Depression will be evaluated using the PHQ-9. ${ }^{34}$ PHQ-9 is based on the diagnostic criteria for major depressive disorder. This scale is widely used as a brief diagnostic tool and measures the severity of depression in both clinical practice and research. The reliability and validity of the scale have been confirmed. ${ }^{42-44}$ This includes nine items on a 4-point scale, with a total score of $0-27$. In this, $0-4,5-9,10-14$ and $15-27$ points indicate a normal, mild, moderate and severe level, respectively. Several studies on mental health during the COVID-19 pandemic have used PHQ-9. ${ }^{10}{ }^{30}$ The Japanese version of PHQ-9 has been reported for its validity and high suitability as a clinical tool for screening and monitoring depression. ${ }^{45} 46$

\section{Insomnia}

Insomnia will be evaluated using the ISI- $7,^{35}$ which consists of seven questions regarding insomnia symptoms in the past 2 weeks: difficulty related to falling asleep, staying asleep and early waking up; satisfaction with sleep pattern; interference in daily work because of sleep problems; noticeability of sleep problems by others and worries regarding sleep problems. Responses are recorded on a 5-point Likert scale ranging from 0 to 4 . The total score ranges from 0 to 28 points, with higher scores indicating higher subjective severity. According to the total score, the patients are classified as 'normal ( $0-7$ points)', 'subthreshold level (8-14 points)', 'moderate insomnia (15-21 points)' and 'severe insomnia (22-28 points)'. The Japanese version of the ISI-7 has been reported to have high reliability and validity. ${ }^{47}$ The Cronbach's $\alpha$ for the ISI-7 Japanese version was 0.84 .

\section{Sense of fear}

Sense of fear will be evaluated using the FCV-19S. ${ }^{36}$ Fear of COVID-19 will be evaluated using 7-items on a 5-point scale: '1: strongly disagree', '2: disagree', '3: neither agree nor disagree', '4: agree', and ' 5 : strongly agree'. The total score is calculated by adding up each item score (ranging from 7 to 35). The higher the score, the greater the fear of COVID-19. FCV-19S has been verified for reliability and validity in many countries ${ }^{48-52}$ since its development, and the Japanese version has been reported to have high reliability and validity. ${ }^{53}$ The Cronbach's $\alpha$ for the FCV19S Japanese version was 0.87 .

\section{Statistical analysis}

Descriptive statistics will be calculated for mental healthrelated items and each measurement item for nursing students. Categorical variables will be summarised using frequencies and proportions. Continuous variables will be presented using means and SD, if normally distributed or medians and IQR, if not normally distributed.

To evaluate the potential risk factors for anxiety, depression, and insomnia symptoms in nursing students, binary logistic regression analysis will be performed; the associations with risk factors will be displayed as ORs and $95 \%$ CI. The binary variables for the binary logistic regression analysis will be developed with the cut-off points being moderate or higher for each scale. A univariate analysis will be performed for the two groups developed at the cut-off points, and a multivariate analysis 
will be performed using the explanatory variables with $p<0.2$. The explanatory variables for the multivariate analysis will be the background information, life satisfaction, fear of COVID-19, and physical activity. $\mathrm{p}<0.05$ will be considered significant. In addition, the correlation between anxiety, depression and insomnia will be investigated. These variables will be compared using the Pearson correlation coefficient.

\section{Sample size}

The prevalence of mental health problems among healthcare workers during the COVID-19 pandemic has been reported to be approximately $30 \%-40 \% .{ }^{9}{ }^{10}$ In nursing students, the prevalence of mental health-related problems is reported to be $30 \%-50 \%{ }^{26}{ }^{27}$ Therefore, considering the prevalence of mental health problems in nursing students to be approximately $30 \%, 1300$ cases would be needed to calculate a $30 \%$ frequency item with a $95 \%$ CI of $5 \%$.

We will perform a logistic regression analysis as a secondary analysis. In logistic regression analysis, the number of samples required is at least 10 times the number of explanatory variables to be included in the prediction model. ${ }^{54}$ We will perform logistic regression analysis with approximately 15 explanatory variables. We calculated the required sample size as $10 \times 15=150$. Thus, the target sample size (1300 nursing students) is estimated to be sufficient for logistic analysis. The total number of students in the eight universities is about 3000 , and we estimate that about $40 \%$ of the students will cooperate, so we expect to receive 1300 responses.

\section{Statistical software}

Statistical analyses will be performed using the EZR statistical software (Saitama Medical Centre, Jichi Medical University, Saitama, Japan) and R (The R Foundation for Statistical Computing, Vienna, Austria). ${ }^{55}$

\section{Patient and public involvement statement}

Patients and the public are not involved in the design, execution, and analysis of the study.

\section{Strengths}

First, this proposed study is the first nationwide crosssectional survey on the assessment of mental health of nursing students in Japan during the COVID-19 pandemic. In addition, the participants of the survey are nursing students studying at universities in the Kanto, Kansai, Chugoku and Kyushu/Okinawa regions, and we believe that the data will be relatively representative of Japan. Furthermore, another strength is the multidimensional assessment of psychological aspects, such as fear of COVID-19. Moreover, out intent to use life satisfaction as well as the GAD-7, PHQ-9, ISI-7, FCV-19S and IPAQ-SF scales to comprehensively assess the mental health of nursing students in Japan, will be an added strength of this study.

\section{Limitation}

A limitation of this study is the expected low participation and response rates, which are inherent in online surveys. To avoid this limitation, the following efforts will be made: First, the announcement with the survey link will be posted on the university web system or portal site system for 2 months, and reminder notices will be sent at least three times starting 2 weeks after the initial announcement to students. Assuming that the participation rate would still be low, we have planned to recruit a total of 3000 nursing students from eight universities to co-operate in the survey for this study. Second, students are most likely to respond to surveys from their smartphones; therefore, to make it as easy as possible for them to answer, we have created a drop-down option to ensure complete and correct responses.

Another possible limitation is that as a self-selection bias, nursing students who do not co-operate with the survey may have more mental health problems than those who do. To avoid this bias, the confidentiality and anonymity of the responses will be guaranteed to the participants at the time of invitation to participate in the survey, and it will be informed that they will not be at a disadvantage regardless of their participation in the survey.

Moreover, because of the cross-sectional nature of the study, misclassification bias and time-related bias may occur. In this study, we intend to investigate anxiety, depression and insomnia using the GAD-7, PHQ-9 and ISI-7 scales, respectively; therefore, the participants will be asked to recall and rate their past 2 weeks. This may lead to underestimation or overestimation of screening due to misclassification. In addition, causality may be reversed if anxiety, depression, or insomnia existed before the COVID-19 pandemic.

\section{ETHICS AND DISSEMINATION}

This study was approved by the Institutional Review Board of Research Ethics committee, College of Nursing Art \& Science and Research Institute of Nursing Care for People and Community, University of Hyogo, Japan (approval no.: 2020F29, approval date: 3 March 2021). If necessary, we plan to obtain ethical approval from the following institutions: Kansai University of Social Welfare, Japanese Red Cross Kyushu International College of Nursing, Okayama Prefectural University, Meio University, Kitasato University and Aichi Medical University. This study was registered before the first participant was enrolled (University Hospital Medical Information Network, Japan (UMIN000044355)). This study will be web based, and all the participants will be instructed to respond after agreeing to the aims of the research.

In order to disseminate the results of the study, we plan to submit the results to a peer-reviewed journal and to present them at local and international conferences to share the results with a wide range of population. Furthermore, the results of this study will be disseminated widely 
at academic conferences in Japan through exchange meetings.

\section{Author affiliations}

${ }^{1}$ College of Nursing Art and Science, University of Hyogo, Akashi, Japan

${ }^{2}$ Faculty of Nursing, Japanese Red Cross Kyushu International College of Nursing,

Munakata, Japan

${ }^{3}$ Department of Nursing, Meio University, Nago, Japan

${ }^{4}$ Faculty of Nursing, Kansai University of Social Welfare, Ako, Japan

${ }^{5}$ Department of Nursing Science, Okayama Prefectural University, Soja, Japan

${ }^{6}$ School of Nursing, Aichi Medical University, Nagakute, Japan

${ }^{7}$ Faculty of Nursing, Kawasaki University of Medical Welfare, Kurashiki, Japan

${ }^{8}$ Faculty of Nursing, National Defense Medical College, Tokorozawa, Japan

${ }^{9}$ School of Nursing, Kitasato University, Sagamihara, Japan

Acknowledgements We are grateful to all the nursing students who participated in the web-based survey and to those who shared their valuable knowledge and experience in planning and designing the survey.We thank Editage (www.editage.jp) for English-language editing. We appreciate the support of SATISTA (https://www. satista.jp/medical/) for the statistical analysis of our data.

Contributors All the authors conceived the study and drafted the original protocol. $\mathrm{JK}, \mathrm{YI}, \mathrm{KK}, \mathrm{YK}$ and TK participated in refining the protocol. JK, YI, HK, YW, TK, SH, YE, $\mathrm{KK}, \mathrm{KH}, \mathrm{YK}, \mathrm{MI}, \mathrm{YN}, \mathrm{SS}, \mathrm{MK}, \mathrm{YK}, \mathrm{MT}$ and MY contributed to developing the survey questionnaires. JK, Yl, SH, KK and YK played a major role in the statistical analyses. All the authors participated in, read and approved the final manuscript.

This work was supported by the Promotion Project of The Next Generation Study, Post-Corona Field.

Competing interests None declared.

Patient consent for publication Not required.

Provenance and peer review Not commissioned; externally peer-reviewed. Not applicable.

Open access This is an open access article distributed in accordance with the Creative Commons Attribution Non Commercial (CC BY-NC 4.0) license, which permits others to distribute, remix, adapt, build upon this work non-commercially, and license their derivative works on different terms, provided the original work is properly cited, appropriate credit is given, any changes made indicated, and the use is non-commercial. See: http://creativecommons.org/licenses/by-nc/4.0/.

\section{ORCID iD}

Jun Kako http://orcid.org/0000-0001-6875-6275

\section{REFERENCES}

1 World Health Organisation. Who coronavirus disease (COVID-19), 2021Dashboard.. Available: https://covid19.who.int/ [Accessed 27 July 2021].

2 Ministry of Health, Labour, and Welfare. Situation report: current situation in Japan.. Available: https://www.mhlw.go.jp/stf/covid-19/ kokunainohasseijoukyou_00006.html [Accessed 27 July 2021].

3 Elbay RY, Kurtulmuș A, Arpacıoğlu S, et al. Depression, anxiety, stress levels of physicians and associated factors in Covid-19 pandemics. Psychiatry Res 2020;290:113130.

4 Huang Y, Zhao N, disorder Ganxiety. Generalized anxiety disorder, depressive symptoms and sleep quality during COVID-19 outbreak in China: a web-based cross-sectional survey. Psychiatry Res 2020;288:112954.

5 Rajkumar RP. COVID-19 and mental health: a review of the existing literature. Asian J Psychiatr 2020;52:102066.

6 Vindegaard N, Benros ME. COVID-19 pandemic and mental health consequences: systematic review of the current evidence. Brain Behav Immun 2020;89:531-42.

7 World Health Organisation. Mental health: strengthening our response, 2018. Available: https://www.who.int/en/news-room/factsheets/detail/mental-health-strengthening-our-response [Accessed 24 October 2021]

8 Pashazadeh Kan F, Raoofi S, Rafiei S, et al. A systematic review of the prevalence of anxiety among the general population during the COVID-19 pandemic. J Affect Disord 2021;293:391-8.

9 Lai J, Ma S, Wang Y, et al. Factors associated with mental health outcomes among health care workers exposed to coronavirus disease 2019. JAMA Netw Open 2020;3:e203976.
10 Vizheh M, Qorbani M, Arzaghi SM, et al. The mental health of healthcare workers in the COVID-19 pandemic: a systematic review. $J$ Diabetes Metab Disord 2020:1967-78.

11 Awano N, Oyama N, Akiyama K, et al. Anxiety, depression, and resilience of healthcare workers in Japan during the coronavirus disease 2019 outbreak. Intern Med 2020;59:2693-9.

12 United Nations Educational,, Scientific and Cultural Organization Influence of COVID-19 situation on education, 2021. Available: https://en.unesco.org/themes/education-emergencies/coronavirusschool-closures [Accessed 27 July 2021]

13 Usher K, Wynaden D, Bhullar N, et al. The mental health impact of COVID-19 on PRE-REGISTRATION nursing students in Australia. Int J Ment Health Nurs 2020;29:1015-7.

14 Natarajan J, Joseph MA. Impact of emergency remote teaching on nursing students' engagement, social presence, and satisfaction during the COVID-19 pandemic. Nurs Forum 2021. doi:10.1111/ nuf.12649. [Epub ahead of print: 07 Sep 2021].

15 Lengetti E, Cantrell MA, DellaCroce N, et al. Learning environment and evidence among professionals and students satisfaction (leaps), experienced during the COVID-19 pandemic. Teach Learn Nurs 2021;16:342-6.

16 Kim JS, Choi JS. Factors influencing emergency nurses' burnout during an outbreak of middle East respiratory syndrome coronavirus in Korea. Asian Nurs Res 2016;10:295-9.

17 Bahçecioğlu Turan G, Özer Z, Çiftçi B. Analysis of anxiety levels and attitudes of nursing students toward the nursing profession during the COVID-19 pandemic. Perspect Psychiatr Care 2021;57:1913-21.

18 Kaplan Serin E, Doğan R. The relationship between anxiety and hopelessness levels among nursing students during the COVID-19 pandemic and related factors. Omega 2021;302228211029144:0030 22282110291.

19 Kuru Alici N, Ozturk Copur E. Anxiety and fear of COVID-19 among nursing students during the COVID-19 pandemic: a descriptive correlation study. Perspect Psychiatr Care 2021. doi:10.1111/ ppc.12851. [Epub ahead of print: 20 May 2021].

20 Köktürk Dalcalı B, Durgun H, Taș AS. Anxiety levels and sleep quality in nursing students during the COVID-19 pandemic. Perspect Psychiatr Care 2021;57:1999-2005.

21 Ashcroft R, Donnelly C, Dancey M, et al. Primary care teams' experiences of delivering mental health care during the COVID-19 pandemic: a qualitative study. BMC Fam Pract 2021;22:143.

22 Gray BM, Vandergrift JL, Barnhart BJ. Changes in stress and workplace shortages reported by U.S. critical care physicians treating coronavirus disease 2019 patients. Crit Care Med 2021;49:1068-82.

23 International Council of Nurses. COVID-19 pandemic one year on: ICN warns of exodus of experienced nurses compounding current shortages, 2021. Available: https://www.icn.ch/news/covid19-pandemic-one-year-icn-warns-exodus-experienced-nursescompounding-current-shortages [Accessed 24 October 2021].

24 Grande RAN, Butcon VER, Indonto MCL, et al. Quality of life of nursing internship students in Saudi Arabia during the COVID-19 pandemic: a cross-sectional study. Int J Afr Nurs Sci 2021;14:100301.

25 Grubic N, Badovinac S, Johri AM. Student mental health in the midst of the COVID-19 pandemic: a call for further research and immediate solutions. Int J Soc Psychiatry 2020;66:517-8.

26 Alsolais A, Alquwez N, Alotaibi KA, et al. Risk perceptions, fear, depression, anxiety, stress and coping among Saudi nursing students during the COVID-19 pandemic. J Ment Health 2021;30:194-201.

27 Zhu $\mathrm{Y}$, Wang $\mathrm{H}$, Wang $\mathrm{A}$. An evaluation of mental health and emotion regulation experienced by undergraduate nursing students in China during the COVID-19 pandemic: a cross-sectional study. Int J Ment Health Nurs 2021;30:1160-9.

28 Santangelo OE, Provenzano S, Armetta F, et al. Prevalence of depressive symptomatology among nursing students during the COVID-19 pandemic. Ann Ig 2021. doi:10.7416/ai.2021.2462. [Epub ahead of print: 06 Jul 2021]

29 Liu S, Xi H-T, Zhu Q-Q, et al. The prevalence of fatigue among Chinese nursing students in post-COVID-19 era. PeerJ 2021;9:e11154.

30 Cénat JM, Blais-Rochette C, Kokou-Kpolou CK, et al. Prevalence of symptoms of depression, anxiety, insomnia, posttraumatic stress disorder, and psychological distress among populations affected by the COVID-19 pandemic: a systematic review and meta-analysis. Psychiatry Res 2021;295:113599.

31 Lopes AR, Nihei OK, Depression NOK. Depression, anxiety and stress symptoms in Brazilian university students during the COVID-19 pandemic: predictors and association with life satisfaction, psychological well-being and coping strategies. PLoS One 2021;16:e0258493. 
32 Xiang M-Q, Tan X-M, Sun J, et al. Relationship of physical activity with anxiety and depression symptoms in Chinese college students during the COVID-19 Outbreak. Front Psychol 2020;11:582436.

33 Spitzer RL, Kroenke K, Williams JBW, et al. A brief measure for assessing generalized anxiety disorder: the GAD-7. Arch Intern Med 2006;166:1092-7.

34 Kroenke K, Spitzer RL, Williams JB. The PHQ-9: validity of a brief depression severity measure. J Gen Intern Med 2001;16:606-13.

35 Morin CM, Belleville G, Bélanger L, et al. The insomnia severity index: psychometric indicators to detect insomnia cases and evaluate treatment response. Sleep 2011;34:601-8.

36 Ahorsu DK, Lin CY, Imani V. The fear of COVID-19 scale: development and initial validation. Int J Ment Health Addict 2020:1-9

37 Craig CL, Marshall AL, Sjöström M, et al. International physical activity questionnaire: 12 -country reliability and validity. Med Sci Sports Exerc 2003;35:1381-95.

38 Murase N, Katsumura T, Ueda C. Validity and reliability of Japanese version of international physical activity questionnaire. Journal of Health and Welfare Statistics 2002;49:1-9.

39 Tang S, Xiang M, Cheung T, et al. Mental health and its correlates among children and adolescents during COVID-19 school closure: the importance of parent-child discussion. J Affect Disoro 2021;279:353-60.

40 Muramatsu K, Miyaoka H, Ueshima K. Validation and utility of a Japanese version of the GAD-7. Japanese Journal of Psychosomatic Medicine 2010;50:166.

41 Doi S, Ito M, Takebayashi Y, et al. Factorial validity and invariance of the 7-item generalized anxiety disorder scale (GAD-7) among populations with and without self-reported psychiatric diagnostic status. Front Psychol 2018;9:9.

42 Löwe B, Kroenke K, Herzog W, et al. Measuring depression outcome with a brief self-report instrument: sensitivity to change of the patient health questionnaire (PHQ-9). J Affect Disord 2004;81:61-6.

43 Spitzer RL, Kroenke K, Williams JB. Validation and utility of a selfreport version of PRIME-MD: the PHQ primary care study. primary care evaluation of mental disorders. patient health questionnaire. JAMA 1999;282:1737-44.
44 Wright AA, Keating NL, Balboni TA, et al. Place of death: correlations with quality of life of patients with cancer and predictors of bereaved caregivers' mental health. $J$ Clin Oncol 2010;28:4457-64.

45 Muramatsu K, Miyaoka H, Kamijima K, et al. The patient health questionnaire, Japanese version: validity according to the miniinternational neuropsychiatric interview-plus. Psychol Rep 2007;101:952-60.

46 Muramatsu K, Miyaoka H, Kamijima K, et al. Performance of the Japanese version of the patient health Questionnaire-9 (J-PHQ-9) for depression in primary care. Gen Hosp Psychiatry 2018;52:64-9.

47 Munezawa T, Morin CM, Inoue Y. Development of the Japanese version of the insomnia severity index (ISI-J). Japanese Journal of Psychiatric Treatment 2009;24:219-25.

48 Sakib N, Bhuiyan A, Hossain S. Psychometric validation of the Bangla fear of COVID-19 scale: confirmatory factor analysis and Rasch analysis. Int J Ment Health Addict2020:1-12.

49 Reznik A, Gritsenko V, Konstantinov V, et al. COVID-19 fear in eastern Europe: validation of the fear of COVID-19 scale. Int J Ment Health Addict 2020:1-6.

50 Soraci P, Ferrari A, Abbiati FA, et al. Validation and psychometric evaluation of the Italian version of the fear of COVID-19 scale. Int $J$ Ment Health Addict 2020:1-10.

51 Tzur Bitan D, Grossman-Giron A, Bloch Y, et al. Fear of COVID-19 scale: psychometric characteristics, reliability and validity in the Israeli population. Psychiatry Res 2020;289:113100.

52 Alyami M, Henning M, Krägeloh CU, et al. Psychometric evaluation of the Arabic version of the fear of COVID-19 scale. Int J Ment Health Addict 2020:1-14.

53 Wakashima K, Asai K, Kobayashi D, et al. The Japanese version of the fear of COVID-19 scale: reliability, validity, and relation to coping behavior. PLoS One 2020;15:e0241958.

54 Peduzzi P, Concato J, Kemper E, et al. A simulation study of the number of events per variable in logistic regression analysis. $J$ Clin Epidemiol 1996;49:1373-9.

55 Kanda Y. Investigation of the freely available easy-to-use software 'EZR' for medical statistics. Bone Marrow Transplant 2013;48:452-8. 